\title{
The Effect of Hachimi-Jio-Gan (Ba-Wei-Di-Huang- Wan) on the Quality of Life in Patients with Peripheral Arterial Disease - A Prospective Study Using Kampo Medicine
}

\author{
Koji Kawago, MD, ${ }^{1}$ Shunya Shindo, PhD, ${ }^{1}$ Hidenori Inoue, PhD, ${ }^{1}$ Junetsu Akasaka, PhD, ${ }^{1}$ \\ Shinya Motohashi, MD, ${ }^{1}$ Go Urabe, PhD, ${ }^{1}$ Masahiro Sato, MD, ${ }^{1}$ Hirotomo Uchiyama, MD, ${ }^{1}$ \\ and Hitoshi Ogino, $\mathrm{PhD}^{2}$
}

\begin{abstract}
Objective: To assess whether Hachimi-jio-gan (HJG), a preparation of Kampo medicine (traditional Japanese medicine), improves quality of life (QOL) in patients with peripheral arterial disease (PAD).

Materials and Methods: Among the patients with PAD being followed in the Department of Cardiovascular Surgery at Tokyo Medical University Hachioji Medical Center, those with intermittent claudication (IC) and in stable condition regarding PAD severity were registered. We registered the patients from April 2014 to March 2015. We administered HJG extract for 6 months to the patients. The primary endpoint was Walking Impairment Questionnaire (WIQ) score, which was approved as an indicator of QOL of the patient with PAD. We assessed WIQ score both before and after administration of the HJG.

Results: We analyzed 14 patients. WIQ items of pain, distance, and speed improved significantly. Furthermore, the median of the total score of WIQ improved significantly from 162.5 points to 308.0 points. All patients showed improvement in the total score and 7 patients out of 14 patients (50\%) showed a remarkably effective improvement in score of more than 100 points.
\end{abstract}

Conclusion: HJG might improve the QOL in patients with IC due to PAD.

Keywords: Hachimi-jio-gan, Ba-Wei-Di-Huang-Wan, intermittent claudication, PAD, cilostazol

${ }^{1}$ Department of Cardiovascular Surgery, Tokyo Medical University Hachioji Medical Center, Tokyo, Japan

${ }^{2}$ Department of Cardiovascular Surgery, Tokyo Medical

University, Tokyo, Japan

Received: December 24, 2015; Accepted: August 5, 2016 Corresponding author: Koji Kawago, MD. Department of Cardiovascular Surgery, Tokyo Medical University Hachioji Medical Center, Tokyo, Japan

Tel: +81-836-22-2260, Fax: +81-836-22-2423

E-mail: kkawagoh@yamanashi.ac.jp

It should be noted that part of this content was presented at the 43rd Annual Meeting of the Japanese Society for Vascular Surgery and the 66th Annual Meeting of the Japan Society for Oriental Medicine.

\section{Introduction}

Intermittent claudication (IC) resulting from peripheral arterial disease (PAD) often limits ability to walk and exercise performance of the patients. Cilostazol is recommended as a medicine for IC. ${ }^{1)}$ Cilostazol improved walking performance with Evidence Level A in patients with IC resulting from PAD. ${ }^{2)}$ However, cilostazol cannot be used for patients with congestive heart failure, which patients with PAD often have. Moreover, some patients cannot continue to take cilostazol due to side effects such as headache, diarrhea, and palpitations.

Hachimi-jio-gan (HJG) is a preparation of traditional Japanese medicine called Kampo medicine. HJG has been used for disorders of the lower part of the body in Japan for a very long time. Otsuka reported a number of case reports in which HJG improved the symptoms of IC resulting from arteriosclerosis. ${ }^{3,4)}$ In addition, HJG was recommended as the first-line Kampo medicine for the treatment of IC resulting from arteriosclerosis in Otsuka's document "Kampo-shinryo-iten." ") "Kamposhinryo-iten" is famous as a dictionary of medical examination and treatment with Kampo medicine. However, until now, no clinical research has shown that HJG improves the symptoms of IC in patients with PAD.

One of the most important indicators for judging effectiveness for treatment of IC is the subjective symptoms indicating the quality of life (QOL) of the patient. ${ }^{1)}$ As quantitative indicators of QOL of patients with PAD, the Walking Impairment Questionnaire (WIQ) is approved internationally ${ }^{1)}$; it evaluates subjective symptoms of the patient. Moreover, the walking ability indicators of asymptomatic walking distance and maximum walking distance correlate with distance and speed score of WIQ.6,7) In addition, there is a correlation between ankle-brachial pressure index (ABI) and WIQ effectiveness. ${ }^{8,9)}$ 
Therefore, we decided to conduct a prospective study to assess whether HJG improves the QOL in patients with PAD.

\section{Materials and Methods}

We used Tsumura HJG extract granules for medical use (TJ-7) as HJG.

\section{Subjects}

Among the patients with PAD being followed in the Department of Cardiovascular Surgery at Tokyo Medical University Hachioji Medical Center, the patients with IC in stable condition as to PAD severity were registered. We registered the patients from April 2014 to March 2015. The definition of PAD in this study was an ankle-brachial pressure index of less than 0.9. Exclusion criteria were as follows: no indication for TJ-7; critical comorbidity (liver disease, kidney disease, heart disease, blood disease, or metabolic disease); receiving or planning to receive medicine under development; and receiving or planning to receive other Kampo preparations. Historically, HJG is contraindicated in patients with clapotement of the lower heart (splashing sound at the epigastric region when tapped). Therefore, we also excluded patients with lower heart clapotement and air-fluid level formation at the stomach on upright abdominal X-rays. In regards to the indications for TJ-7, we followed the attached document, namely, nephritis, diabetes, impotence, sciatica, lumbago, beriberi, bladder catarrh, prostatic hypertrophy, and hypertension.

All patients provided written informed consent. This study protocol was approved by the ethics committee of Tokyo Medical University Hachioji Medical Center (receipt number: H-6), and registered in the University Hospital Medical Information Network Clinical Trials Registry (identification number: UMIN000017332).

\section{Study protocol}

We administered TJ-7 as $7.5 \mathrm{~g}$ divided into three portions for oral administration before or between meals for 6 months. The primary endpoint was the Japanese Version WIQ. ${ }^{10)} \mathrm{ABI}$ and skin perfusion pressure (SPP) were measured as indicators of ischemia of the lower extremities. We assessed the score of WIQ, ABI, and SPP both before and after administration of the TJ-7. We did not give any new interventions to the selected patients for 6 months, including therapeutic exercise, revascularization, or non-smoking instruction. In addition, we did not change any medicine other than TJ-7.

\section{Statistical analysis}

All data are expressed as the median (interquartile range) unless otherwise specified. Differences in the WIQ score,
Table 1 Background factors and risk factors of the patients with peripheral arterial disease

\begin{tabular}{lc}
\hline Age $($ Mean \pm SD) & $73.3 \pm 4.7$ \\
Weight $(\mathrm{kg})($ Mean \pm SD) & $57.7 \pm 7.8$ \\
BMI $\left(\mathrm{kg} / \mathrm{m}^{2}\right)$ (Mean \pm SD) & $23.5 \pm 3.9$ \\
Sex & 9 \\
Male & 5 \\
Female & \\
Limb & 3 \\
Right & 2 \\
Left & 9 \\
$\quad$ Both & \\
Cigarette smoking & 5 \\
$\quad$ Never & 5 \\
$\quad$ Previous & 4 \\
$\quad$ Current & 12 \\
Hypertension & 7 \\
Diabetes mellitus & 2 \\
Hyperlipidemia & \\
\hline SD: standard deviation; BMl: body mass index; \\
Limb: ischemic limb
\end{tabular}

ABI, and SPP between after HJG administration and baseline were assessed using the paired $t$-test. $P<0.05$ was the threshold for statistical significance. All analyses were performed using IBM SPSS Statistics Version 22.

\section{Results}

\section{Patient characteristics}

A total of 19 patients were registered in this study; 5 patients dropped out. Table 1 shows background factors of 14 remaining patients. All of them had a diagnosis of arteriosclerosis obliterans (ASO) and they were all at class 2 in the Fontaine classification.

\section{Changes in WIQ items}

Table 2 shows ABI, SPP, and WIQ evaluation items for the 14 patients with PAD. As to ABI and SPP, no improvements were found by administration of the TJ-7; the SPP of the dorsal side of the foot actually significantly decreased $(P=$ 0.011) after administration of TJ-7. The WIQ items of pain, distance, and speed improved significantly $(P<0.05)$ after administration of TJ-7, although the WIQ item of climbing did not $(P=0.068)$. The total score of WIQ improved significantly from $162.5(32.0-250.0)$ points to $308.0(205.0-387.5)$ points $(P<0.05)$ after administration of TJ-7 (Fig. 1 and Table 2). All patients showed improvement in the total score and 7 patients out of 14 patients $(50 \%)$ showed a remarkably effective improvement in score of more than 100 points after administration of TJ-7.

\section{Adverse events}

Five patients out of 19 patients $(26.3 \%)$ dropped out. The details of reasons for dropping out are shown in Table 3. 

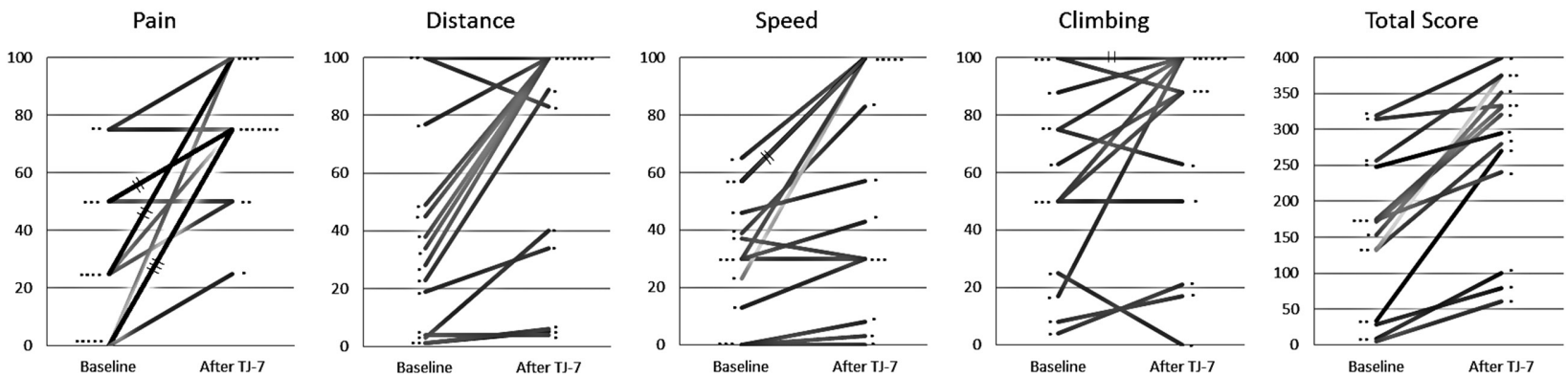

Fig. 1 Changes in value of Walking Impairment Questionnaire (WIQ) score for each patient. One point represents one patient with peripheral arterial disease. Overlapped lines are expressed by bars on the line: for example, there are two patients whose pain score changed from 50 points to 75 points. The WIQ items of pain, distance, speed, and the total score of WIQ improved significantly $(P<0.05)$ after administration of TJ-7. TJ-7, Tsumura Hachimi-jio-gan extract granules for medical use.

Table 2 A comparison of ABI, SPP, and WIQ between baseline and after administration of TJ-7 for 6 months

\begin{tabular}{lcccr}
\hline & Baseline & After TJ-7 & Absolute change & $P$ value \\
\hline ABI & $0.69(0.48-0.81)$ & $0.73(0.51-0.83)$ & $0.02(-0.05-0.09)$ & 0.463 \\
SPP $(\mathrm{mmHg})$ & & & & \\
$\quad$ Dorsal & $52.5(39.5-63.0)$ & $43.0(35.0-50.0)$ & $-9.0(-16.0-2.50)$ & 0.011 \\
$\quad$ Plantar & $51.0(37.5-57.0)$ & $46.0(38.0-57.0)$ & $-6.0(-13.5-5.0)$ & 0.224 \\
WIQ & & & & \\
Pain & $25.0(0.0-50.0)$ & $75.0(68.8-100.0)$ & $37.5(25.0-75.0)$ & $<0.001$ \\
Distance & $31.0(3.8-56.0)$ & $94.5(27.0-100.0)$ & $30.0(3.0-63.0)$ & 0.002 \\
Speed & $30.0(9.8-48.8)$ & $50.0(24.5-100.0)$ & $15.0(2.3-43.3)$ & 0.003 \\
Climbing & $56.5(23.0-91.0)$ & $88.0(42.8-100.0)$ & $10.5(-3.0-28.3)$ & 0.068 \\
$\quad$ Total score & $162.5(32.0-250.0)$ & $308.0(205.0-387.5)$ & $105.0(53.8-166.0)$ & $<0.001$ \\
\hline
\end{tabular}

Values are expressed as the median (interquartile range). ABI: ankle-brachial pressure index; SPP: skin perfusion pressure; WIQ: Walking Impairment Questionnaire; TJ-7: Tsumura Hachimi-jio-gan extract granules for medical use; Dorsal: dorsal side of the foot; Plantar: plantar side of the foot

Table 3 Backgrounds of the patients who dropped out and the causes of dropping out

\begin{tabular}{|c|c|c|c|c|c|c|c|c|c|c|}
\hline \multirow{2}{*}{ Case } & \multirow{2}{*}{ Age } & \multirow{2}{*}{ Sex } & \multirow{2}{*}{ Limb } & \multicolumn{2}{|c|}{$A B I$} & \multicolumn{4}{|c|}{ WIQ } & \multirow{2}{*}{$\begin{array}{c}\text { The cause of the } \\
\text { dropping out }\end{array}$} \\
\hline & & & & Right & Left & Pain & Distance & Speed & Climbing & \\
\hline 1 & 81 & Male & Both & 0.71 & 0.68 & 50 & 5 & 7 & 42 & Digestive symptoms \\
\hline 8 & 81 & Male & Left & 0.93 & 0.83 & 100 & 100 & 100 & 100 & Cardiac insufficiency \\
\hline 12 & 88 & Male & Both & 0.64 & 0.66 & 0 & 14 & 17 & 17 & $\begin{array}{l}\text { Inguinal hernia } \\
\text { strangulation }\end{array}$ \\
\hline 16 & 72 & Male & Both & 0.70 & 0.65 & 0 & 9 & 15 & 100 & $\begin{array}{l}\text { Aggravation of low } \\
\text { back pain }\end{array}$ \\
\hline 19 & 73 & Male & Right & 0.60 & 1.11 & 50 & 100 & 30 & 100 & $\begin{array}{l}\text { Unidentified } \\
\text { exanthem }\end{array}$ \\
\hline
\end{tabular}

The values of $A B I$ and WIQ were measured before administration of Tsumura Hachimi-jio-gan extract granules for medical use. Limb: ischemic limb;

$A B I$ : ankle-brachial pressure index; WIQ: Walking Impairment Questionnaire

In Case 1, the patient had lung surgery at 2 months after starting administration. Then, the patient had gastrointestinal symptoms at 4 months after starting administration. At that time, the patient demonstrated air-fluid level formation at the stomach on upright abdominal X-rays and clapotement of the lower heart for the first time. In case 19 , the patient got a rash after starting administration. Although we received a comment from a dermatologist that the cause of the rash was less likely to be HJG, the patient requested stoppage of HJG. As for the other three cases, the patients themselves requested stoppage of $\mathrm{HJG}$ due to the progression of other diseases.

\section{Discussion}

HJG is written as the name of Ba-Wei-Di-Huang-Wan (Eight-Ingredient Pill with Rehmannia) in a classic clinical book of traditional Chinese medicine called "Jin 
Gui Yao Lue (Essential Prescriptions from the Golden Cabinet)," which was written by Zhang Zhongjing (150-219) at the end of the Eastern Han dynasty. The name is derived from the eight kinds of herb materials; Rehmanniae radix (jio), Corni fructus (sanshuyu), Dioscoreae rhizoma (san'yaku), Alismatis rhizoma (takusya), Hoelen (bukuryo), Paeoniae moutan cortex (botanpi), Cinnamomi cortex (keishi), and Aconiti rhizoma (bushi). One of the effects of HJG is on the circulatory system. HJG improved the vascular elasticity rate of the aortic wall in cases of experimental arteriosclerosis in the rat. ${ }^{11)}$ HJG also suppressed blood pressure elevation in metyrapone-treated heat-loaded high blood pressure rats (MHR). ${ }^{12)}$ In addition, HJG had a dose-dependent hypotensive effect on high sodium-induced high blood pressure rats. ${ }^{13)}$ Unfortunately, there is no confirmation for HJG as to the peripheral circulation-improving effect in the lower extremities. However, regarding Gosyazinki-gan (which includes two kinds of herbal drug, namely, Achyranthis radix [goshitsu] and Plantaginis semen [shazenshi] seed into HJG), Shikano et al. reported on improvement of skin blood flow and elevation of skin temperature in patients with diabetes mellitus. ${ }^{14,15)}$ Regarding individual herbal drugs, the antiplatelet action of Paeoniae moutan cortex and Cinnamomi cortex ${ }^{16,17)}$ and analgesic action of Aconiti rhizoma ${ }^{18)}$ have been pointed out; these are assumed to be some of the mechanisms to explain that HJG improved some of the WIQ items. However, it is not clear how much contribution the HJG components have in the whole prescription, which is composed of eight herbal drugs and also contains a number of trace substances. Kampo preparations are composed of several herbal drugs. The medical effect of Kampo preparations cannot be explained by the total of each individual herbal drug action.

As a kampo preparation for patients with PAD, Tokishigyakuka-goshuyu-shokyoto (TSGST) is also sometimes used. Jyojima et al. administered cilostazol together with TSGST extract for 3 months for the patients with IC resulting from PAD; using a treadmill test, they found significant improvement in asymptomatic walking distance and maximum walking distance as compared with those before administration. ${ }^{19)}$ There are some reports of other kampo preparations, which improved symptoms of PAD other than HJG or TSGST. However, these were not clinical studies.

Regensteiner analyzed retrospectively six Phase 3, multicenter, double-blind, placebo-controlled, parallel-group, randomized studies to assess whether cilostazol improves treadmill performance and QOL in patients with IC resulting from PAD. Regensteiner's meta-analysis concluded that cilostazol not only improved objective walking distance, but also WIQ scores. ${ }^{20)}$ All four items in the WIQ
Table 4 A comparison of improvements in Walking Impairment Questionnaire score in patients with peripheral arterial disease between TJ-7 in our research and cilostazol in Regensteiner's report ${ }^{19)}$

\begin{tabular}{lccc}
\hline Score & Baseline & Follow-up & $\begin{array}{c}\text { Absolute } \\
\text { change }\end{array}$ \\
\hline Pain & & & \\
$\quad$ Placebo & $38 \pm 25$ & $47 \pm 27$ & $9 \pm 28$ \\
$\quad$ Cilostazol & $41 \pm 24$ & $55 \pm 27$ & $15 \pm 28$ \\
$\quad$ TJ-7 & $28.6 \pm 27.5$ & $75.0 \pm 21.9$ & $46.4 \pm 32.3$ \\
Distance & & & \\
Placebo & $29 \pm 26$ & $35 \pm 29$ & $6 \pm 25$ \\
Cilostazol & $32 \pm 27$ & $43 \pm 32$ & $11 \pm 27$ \\
TJ-7 & $37.3 \pm 34.2$ & $68.6 \pm 40.8$ & $31.3 \pm 30.4$ \\
Speed & & & \\
Placebo & $37 \pm 27$ & $40 \pm 27$ & $3 \pm 26$ \\
Cilostazol & $39 \pm 28$ & $47 \pm 30$ & $8 \pm 26$ \\
TJ-7 & $30.5 \pm 21.7$ & $56.0 \pm 40.1$ & $25.5 \pm 26.7$ \\
Climbing & & & \\
Placebo & $47 \pm 33$ & $49 \pm 33$ & $2 \pm 30$ \\
Cilostazol & $49 \pm 33$ & $55 \pm 35$ & $6 \pm 28$ \\
TJ-7 & $57.5 \pm 34.2$ & $72.5 \pm 36.0$ & $15.0 \pm 28.2$ \\
\hline
\end{tabular}

Values are expressed as the mean \pm standard deviation. TJ-7: Tsumura Hachimi-jio-gan extract granules for medical use

showed significant improvement in scores compared with placebo after administration for 3 to 6 months. Therefore, cilostazol was recommended for patients with IC resulting from PAD. Table 4 shows a comparison of WIQ scores between our results obtained from TJ-7 and Regensteiner's results obtained from cilostazol. Values in our research are expressed as the mean \pm standard deviation for comparison with Regensteiner's results. Absolute changes by TJ-7 exceeded those by cilostazol on all items of WIQ.

In addition, the mixed effect of revascularization and rehabilitation (MERR) might also improve all the items within WIQ scores and the total WIQ score of the patients with PAD. According to a graph in Kawasaki's report, ${ }^{21)}$ MERR increased the total WIQ score of the patients with non-necrosis and amputation of toes resulting from PAD (NA group) from about 225 points to about 320 points (absolute change is about 95 points). The variations of the WIQ scores of the NA group by MERR were also described in another graph: left foot pain scores were from about 60 to about 85 , right foot pain scores were about 60 to about 80 , walking distance scores were from about 45 to about 70 , speed scores were from about 20 to about 30, and climbing scores were from about 40 to about 60 . In our research, HJG extract increased the total WIQ score of the patient with PAD from 153.9 points to 272.1 points (absolute change is 118.3 points). The total score of WIQ should be interpreted carefully because the pain score has a high power. Only a one-rank change of pain sensation equals a 25 -point change in the score. Our research appears to have larger changes in not only the pain score, but also the 
distance score and the speed score than Kawasaki's study. However, the improvement especially in pain score of WIQ by administrating HJG is significant in comparison with that by administrating cilostazol in Regensteiner's report and by MERR in Kawasaki's report; the absolute changes in pain score of WIQ by HJG, cilostazol, and MERR were 46.4, 15, and 20-25, respectively. Therefore, we thought that the analgesic effect of HJG might be an advantageous effect on improving the scores of WIQ. Regarding the analgesic effect of HJG, there are some reports about walking distance and the symptoms of IC among the patients with lumbar spinal stenosis. ${ }^{22)}$

From the above, our research offers that HJG might improve the QOL in patients with IC due to PAD as well as cilostazol or MERR. Furthermore, particularly in the distance, climbing, and total score of WIQ after administration of TJ-7, the medians are larger than the means (Table 2 and 4). There were no differences between the means and the medians at baseline. This means that many patients reached the full score in those items as shown in Fig. 1. Therefore, the mean values might have shown our results with underestimations by this ceiling effect. However, we cannot simply compare between the variation in WIQ scores of our results and those of Regensteiner's report or those of Kawasaki's report, because research design and background of the patients in their reports were different from our research. In Kawasaki's report, 6 patients were at class 3 in the Fontaine classification in all 32 patients of the NA group. For the next stage, we think we need to prove that HJG improves not only the WIQ scores, but also objective walking distance of patients with PAD; this is because WIQ seems too limited to evaluate the patients administered HJG as shown in our research.

Regarding safety, 5 of 19 patients dropped out and 2 of these 5 patients $(10.5 \%)$ had digestive symptoms or rash. These symptoms are well known as side effects of HJG. In Case 1, we thought the cause of the symptoms might be the deterioration of the general condition of the body resulting from lung surgery. In Case 19, we thought the cause of the rash less likely to be HJG, agreeing with the opinion of the dermatologist. However, we cannot deny that those symptoms are side effects of HJG. Therefore, we think administration of HJG must be done only to the patient in a generally stable condition.

\section{Conclusion}

HJG might improve the QOL in patients with IC due to PAD as well as cilostazol or the mixed effect of revascularization and rehabilitation. However, HJG should be administered only to the patient in a generally stable condition.

\section{Disclosure Statement}

The authors declare no conflict of interest.

\section{Author's Contribution}

Study conception: KK, SS

Data collection: KK, SS, HI, JA, SM, GU, MS, HU, $\mathrm{HO}$

Analysis: KK

Investigation: $\mathrm{KK}$

Writing: KK

Critical review and revision: all authors

Final approval of the article: all authors

Accountability for all aspects of the work: all authors

\section{References}

1) Norgren L, Hiatt WR, Dormandy JA, et al. Inter-Society consensus for the management of peripheral arterial disease (TASC II). J Vasc Surg 2007; 45: S5-67.

2) Society for Vascular Surgery Lower Extremity Guidelines Writing Group, Conte MS, Pomposelli FB, et al. Society for Vascular Surgery practice guidelines for atherosclerotic occlusive disease of the lower extremities: management of asymptomatic disease and claudication. J Vasc Surg 2015; 61: 2S-41S.

3) Otsuka K. Shukindo Experience Record (4). Journal of Kampo Medicine, 1970; 17: 281-4. (in Japanese)

4) Otsuka K. Hachimi-gan for Intermittent Claudication. Otsuka K Collected Works Vol. 4. Shun Yo Do. Tokyo; 1980; 17. (in Japanese)

5) Otsuka K, Yakazu D, Shimizu T. Kampo Shinryo Iten. 6th ed. Tokyo: Nanzando 2007; 90. (dictionary of medical examination and treatment with Kampo medicine in Japanese)

6) Myers SA, Johanning JM, Stergiou N, et al. Claudication distances and the Walking Impairment Questionnaire best describe the ambulatory limitations in patients with symptomatic peripheral arterial disease. J Vasc Surg 2008; 47: 550-5.

7) Verspaget M, Nicolaï SP, Kruidenier LM, et al. Validation of the Dutch version of the Walking Impairment Questionnaire. Eur J Vasc Endovasc Surg 2009; 37: 56-61.

8) Matsuo H, Shigematsu H. Effects of the 5-HT2A antagonist sarpogrelate on walking ability in patients with intermittent claudication as measured using the walking impairment questionnaire. Ann Vasc Dis 2008; 1: 102-10.

9) Feinglass J, McCarthy WJ, Slavensky R, et al. Effect of lower extremity blood pressure on physical functioning in patients who have intermittent claudication. The Chicago Claudication Outcomes Research Group. J Vasc Surg 1996; 24: 503-11; discussion 511-2.

10) Ikeda S, Kobayashi M, Shigematsu H, et al. Development of the Japanese Version of WalkingImpairment 
Questionnaire(WIQ). J Jpn Coll Angiol 2005; 45: 233-40. (in Japanese with English abstract)

11) Amagaya S, Umeda M, Ogihara Y. Analysis of Kampo: No. 29: Effect of Kampo on experimental arteriosclerosis. J Trad Med 1986; 3: 338-9. (in Japanese)

12) Tei S, Arakawa K, Hayashi R. Effect of Kampo medicines on the process of experimental hypertention. (No. 12). J Trad Med 1989; 6: 416-7. (in Japanese)

13) Uehara Y, Omata M. On protective effects of Hachimi-jiogan for blood pressure, cardiovascular and renovascular systems and glomerular disorder in salt sensitive hypertension model rats (Dahl). Prog Med 1994; 14: 1761-6. (in Japanese)

14) Shikano M, Sobajima H. Effect of Goshajinkigan on skin blood flow in diabetes mellitus patient. J Trad Med 1988; 5: 378-9. (in Japanese)

15) Shikano M, Oki Y, Aoki T. Vasodilation effects of Goshajinki-gan on diabetic patients. (comparison with PGE1). J Trad Med 1991; 7: 442-3. (in Japanese)

16) Kubo M, Matsuda H, Matsuda R. Study of Botanpi (No. 8) about antithrombotic action (No. 2). Japan. J Pharmacog 1984; 38: 307-12. (in Japanese)

17) Matsuda H, Matsuda R, Fukuda S, et al. Antithrombic actions of $70 \%$ methanolic extract and cinnamic aldehyde from cinnamomi cortex. Chem Pharm Bull 1987; 35: 1275-80.

18) Takizawa Y, Isono T, Suzuki Y, et al. [Analgesic effects of Tsumura-shuuji-bushi-matsu and mesaconitine]. Nippon Yakurigaku Zasshi 1992; 100: 307-16. (in Japanese)

19) Jyojima K. The Clinical Effectiveness of Traditional Japanese Harbal-medicine, Tokishigyakukagoshuyushyokyoto for Vascular Intermittent Claudication. Kampo Med 2011; 62: 529-36. (in Japanese with English abstract)

20) Regensteiner JG, Ware JE, McCarthy WJ, et al. Effect of cilostazol on treadmill walking, community-based walking ability, and health-related quality of life in patients with intermittent claudication due to peripheral arterial disease: meta-analysis of six randomized controlled trials. J Am Geriatr Soc 2002; 50: 1939-46.

21) Kawasaki M, Takahashi Y, Kawakatsu H, et al. The Evaluation of Walking Impairment Questionnaire with Rehabilitation after Revascularization in Peripheral Arterial Disease. J Jpn Coll Angiol 2012; 52: 13-8. (in Japanese with English abstract)

22) Hayashi Y, Saito E, Takahashi O. Usefulness of Hachimijio-gan for lumbar spinal canal stenosis. Geriatr Med 1994; 32: 585-91. 